\title{
Nuclear Masses, Chaos, and the Residual Interaction
}

\author{
A. Molinari \\ Dipartimento di Fisica Teorica dell' Universita di Torino \\ and Instituto Nationale di Fisica Nucleare, Sezione di Torino, \\ Torino, Italy \\ H. A. Weidenmüller \\ Max-Planck-Institut für Kernphysik, Heidelberg, Germany
}

August 5, 2018

Corresponding author: Hans A. Weidenmüller, Max-Planck-Institut für

Kernphysik, P. O. Box 102980, 69029 Heidelberg, Germany

Telephone: 49/6221/516291 FAX: 49/6221/516602

email: Hans.Weidenmueller@mpi-hd.mpg.de

\begin{abstract}
We interpret the discrepancy between semiempirical nuclear mass formulas and actual nuclear masses in terms of the residual interaction. We show that correlations exist among all binding energies and all separation energies throughout the valley of stability. We relate our approach to chaotic motion in nuclei.
\end{abstract}

Keywords: Binding energies, separation energies, chaos, residual interaction. Pacs: 02.50.-r, 05.40.-a, 75.10.Nr

Motivation. The masses of atomic nuclei are keys to the understanding of many physical and astrophysical processes. For this reason it is important to construct reliable theoretical models for the values of the nuclear masses or, equivalently, of the binding energy $B(A)$ as function of mass number $A$. (For simplicity of notation we suppress the additional dependence on proton number $Z$ ). This function is also needed to predict the masses of (as yet) unknown nuclei.

The standard approach to a global modelling of the function $B(A)$ starts out from the liquid-drop model for the nucleus and considers in addition shell corrections as well as corrections due to pairing. The resulting semi-empirical mass formula contains about 30 parameters and is fitted to a large number of data. Years of painstaking work have culminated in a best fit [1] which reproduces the data points very well albeit not exactly. The overall difference between that best fit and the actual data is of the order of $0.5 \mathrm{Mev}$, with 
a tendency to become smaller for heavier nuclei. That figure, although very small in comparison with the total binding energy, is not negligible. Other approaches [2, 3] lead to similar differences.

Bohigas and Leboeuf [4 have suggested that the discrepancy can be attributed to the fact that the nuclear dynamics is partly chaotic. Using the semiclassical approximation and Berry's result [5] for the form factor of the two-point correlation function, these authors have estimated the contribution to the variance of the binding energy due to chaotic single-particle motion. For the square root of the variance, the estimate yields

$$
\sigma=\frac{2.78}{A^{1 / 3}} \mathrm{MeV} .
$$

Like the shell-correction employed in the best-fit procedure of Ref. 1], Eq. (1) is derived in the framework of the single-particle picture and is, thus, based upon a mean-field description. However, the authors of Ref. 4] state that this equation "presumably gives an estimate of the mass fluctuations from neglected many-body effects". When plotted versus mass number, the result (1) is in good agreement with the observed discrepancy except for the lightest nuclei, see Fig. 1 of Ref. [4.

The work of Ref. 4 has led to a lively discussion in the community. In this letter, we wish to address some of the points that have come up in the discussion. Our point of view differs from that of Ref. 44 in that we interpret the discrepancy in terms of many-body effects due to the residual interaction of the shell model and explore the consequences of such an interpretation. We are primarily interested in the conceptual implications of such an approach. Manybody effects have been addressed in a number of publications, see, for instance, Refs. 6, 7, 8, 9]. We discuss some of these works below.

The Two-Body Random Ensemble. At the outset, we have to specify the residual interaction. We assume that the residual interaction is of two-body form. We do so only to have a specific model within which we can work. We are aware of the fact that there is evidence for weak three-body interactions in nuclei [10]. It will be seen that allowing for three-body forces will not affect our conclusions.

Within every major shell, the residual two-body interaction is specified in terms of a set of two-body matrix elements. All possible two-body interactions are taken into account by considering the two-body matrix elements as Gaussian-distributed random variables. This defines the two-body random ensemble (TBRE) of the shell model [11, 12]. The TBRE has the advantage of leading to generic statements (valid for almost all two-body interactions). This is useful since not all of the two-body matrix elements of the residual interaction of the shell model are precisely known either empirically or theoretically, especially in medium-weight and heavy nuclei, and for higher-lying shells in any nucleus. Moreover, the use of the TBRE allows us to estimate variances of observables and, thus, the range within which values of the observables will vary as we vary the parameters of the residual interaction or, equivalently, to 
estimate the uncertainty of the observables due to incomplete knowledge of the residual interaction.

If one applies the TBRE to the calculation of binding energies (more precisely: to estimate corrections to the semi-empirical mass formula as employed in Ref. [1), the binding energies will vary from one realization of the TBRE to the next. Technically speaking, the binding energies become random variables. Again, their variances express our uncertainty about the residual interaction. We use these variances as a measure of the deviations from empirical values of the binding energies that are expected to occur in semi-empirical mass formulas which neglect parts or all of the residual interaction. (The actual formula used in Ref. 1] does not disregard the residual interaction altogether but does take into account the pairing force and the symmetry energy. The version of the TBRE used here must take this fact into account by keeping fixed the corresponding two-body matrix elements, and by considering as random only the remainder).

Binding Energies and Separation Energies. We discuss the application of the TBRE to binding energies. We first recall the connection between nuclear binding energies and nucleon separation energies. We denote the separation energy of the least-bound nucleon in nucleus $A$ (which is either a neutron or a proton) by $S(A)$, again omitting for simplicity the dependence of $S$ on $Z$. We can completely decompose the nucleus $A$ into its constituent nucleons by removing one nucleon after the other. Thus we have the identity

$$
B(A)=\sum_{j=2}^{A} S(j) .
$$

Likewise, since after removal of $k$ nucleons we reach the nucleus with mass number $A-k$ (with $k$ integer), we also have

$$
B(A)=B(A-k)+\sum_{j=A-k+1}^{A} S(j) .
$$

Given the initial nucleus with mass number $A$ and proton number $Z$, the $S(j)$ in Eqs. (2) and (3) are uniquely specified by the requirement that we always remove the least-bound nucleon. With this understanding, Eqs. (2) and (3) hold for all nuclei (and not only for those in the bottom of the valley of stability) and for all values of $k$. More equations of the same type are obtained when we allow for the removal of the least-bound proton or least-bound neutron when that is not the least-bound nucleon. Hence, equations of the type of Eqs. (2) and (3) constitute a dense network of identities that hold throughout the mass valley.

It was stated above that as we apply the TBRE to calculate corrections to the binding energies predicted by the model of Ref. [1], such binding energies become random variables. The same holds for the nucleon separation energies. For clarity these are denoted by the letters $\mathcal{B}$ and $\mathcal{S}$, respectively. The random 
variables $\mathcal{B}(A)$ and $\mathcal{S}(j)$ must obey equations of the type (2) and (3), since by the very definition of the binding energy and the separation energy, every realization of $\mathcal{B}(A)$ and $\mathcal{S}(j)$ in the framework of the TBRE must obey these equations. Thus,

$$
\begin{gathered}
\mathcal{B}(A)=\sum_{j=2}^{A} \mathcal{S}(j), \\
\mathcal{B}(A)=\sum_{j=A-k+1}^{A} \mathcal{S}(j)+\mathcal{B}(A-k) .
\end{gathered}
$$

Correlations. We recall that the deviations of the data from the empirical mass formula decrease with increasing values of mass number $A$, see Eq. (10). We now show that this fact combined with Eqs. (41) and (5) implies strong correlations between the random variables $\mathcal{B}(A)$ and $\mathcal{S}(A)$ for all values of $A$. We recall that stochastic contributions to these variables arise from the TBRE. If we assume that all variables $\mathcal{B}(A)$ and $\mathcal{S}(A)$ are uncorrelated, it follows from Eq. (41) that

$$
\operatorname{Var}(\mathcal{B}(A))=\sum_{j=2}^{A} \operatorname{Var}(\mathcal{S}(j))
$$

The variance is, by definition, positive semidefinite. Therefore, Eq. (6) implies that $\operatorname{Var}(\mathcal{B}(A))$ is a non-decreasing function of $A$. (That same conclusion was drawn already in Ref. [13] albeit in the framework of a specific model. Here we establish it in full generality). This result is in striking contrast to the monotonic decrease of the discrepancy with increasing mass number displayed by the data and by Eq. (1). We conclude that there exist strong correlations between binding energies and separation energies.

Allowing for such correlations to exist, we arrive at a modified form of Eq. (6). With the covariance of two random variables $X$ and $Y$ defined as $\operatorname{Cov}(X Y)=\overline{X Y}-\bar{X} \bar{Y}$, we have

$$
\operatorname{Var}(\mathcal{B}(A))=\sum_{j=2}^{A} \operatorname{Var}(\mathcal{S}(j))+\sum_{i \neq j ; i, j=2}^{A} \operatorname{Cov}(\mathcal{S}(i) \mathcal{S}(j)) .
$$

In order to attain the observed monotonic decrease of the discrepancy with mass number, the sum of the covariances must be negative semidefinite for all values of $A$ and cannot vanish identically. This definitely implies the existence of correlations among the $\mathcal{S}(j)$ 's. Given this fact, we expect correlations to exist also among binding energies pertaining to different values of $A$. Indeed, Eq. (4) implies

$$
\operatorname{Cov}(\mathcal{B}(A) \mathcal{B}(A-k))=\sum_{j=2}^{A-k} \operatorname{Var}(\mathcal{S}(j))+\sum_{j_{1}=2}^{A} \sum_{j_{2}=2}^{A-k}\left(1-\delta_{j_{1} j_{2}}\right) \operatorname{Cov}\left(\mathcal{S}\left(j_{1}\right) \mathcal{S}\left(j_{2}\right)\right)
$$


This expression is, in general, different from zero. In contrast to the case of the separation energies we have, however, no firm empirical evidence in the present framework that these correlations do indeed exist. They may vanish accidentally for all values of $A$. Even in this case we expect correlations of higher order than the second to differ from zero. Indeed, it takes a very special form of the probability density of random variables connected by linear relations like Eq. (44) to be uncorrelated. We conclude that within the framework of the TBRE, all binding energies and all separation energies throughout the mass valley are correlated.

Discussion. From the point of view of nuclear physics, this result is not terribly surprising. It mirrors the fact that a change of the residual interaction within a major shell will affect the binding energies and the separation energies of all nuclei pertaining to that shell. As we cross the boundary in mass number separating major shells, the dominant two-body matrix elements of the residual interaction change, and we might expect a weakening of the correlations. Our result shows no trace of such an effect, and this is probably its most surprising feature from the nuclear physics point of view.

Unfortunately, we cannot draw any conclusions about the range of the correlations. Still, the following model is instructive. We assume that the correlations between the separation energies are minimal and restricted to nearest neighbors only so that $\operatorname{Cov}\left(\mathcal{S}\left(j_{1}\right) \mathcal{S}\left(j_{2}\right)\right) \propto \delta_{j_{1}, j_{2} \pm 1}$. For the range of the correlations, this is a worst-case scenario. Our assumption is consistent with Eq. (7) provided all these covariances are negative definite. By a suitable choice of the values of the covariances, it is possible to account for the monotonic decrease of $\sigma$ with $A$ as postulated in Eq. (11). However, even that model implies long-range correlations between the binding energies. Indeed, under the assumptions of the model, the right-hand side of Eq. (8) becomes equal to $\operatorname{Var}(\mathcal{B}(A-k))+\operatorname{Cov}(\mathcal{S}(A-k+1) \mathcal{S}(A-k)$. Thus, the covariances of $\mathcal{B}(A-k)$ with $\mathcal{B}(A)$ have for all values of $A$ the same values! We have not been able to design a model which would satisfy Eqs. (7) and (8) with short-range correlations between all $\mathcal{S}$ 's and all $\mathcal{B}$ 's. From the point of view of the TBRE, we expect the correlations to extend over a range of mass numbers given by the range of major shells, see Ref. [14].

Statistical fluctuations of nuclear spectra are commonly described not in the framework of the TBRE but rather in the framework of the Gaussian orthogonal ensemble of random matrices (GOE). From the point of view of canonical random-matrix theory, the existence of long-range correlations is unexpected. Indeed, at present there exists no conceptual framework within the GOE in which the existence of such correlations could be accommodated. Thus, the data point to a further limitation of canonical random-matrix theory. Earlier work on $s d$-shell nuclei [14] has revealed such limitations in a more restricted framework by proving the existence of correlations between levels in the same nucleus carrying different quantum numbers and between levels in different nuclei belonging to the same major shell.

What is the relation between our point of view - which blames the discrepancy between data and the nuclear mass formula on the residual interaction - 
and that taken in Ref. 4] - which puts the blame on order-chaos coexistence? We identify chaos in nuclei with the existence of spectral fluctuations of the Wigner-Dyson type. This statement applies to levels of fixed spin and parity within the same nucleus. In the following three paragraphs we present arguments to the effect that such spectral fluctuations are always due to the residual interaction. Given this fact, we conclude that the two points of view are not fundamentally different especially in view of the remark 4] that Eq. (1) "presumably gives an estimate of the mass fluctuations from neglected many-body effects".

To justify our view that the residual interaction is the agent responsible for chaos in nuclei, we consider first spherical nuclei. Here the single-particle motion is regular and does not lead to level sequences which obey Dyson-Mehta statistics. This changes when the residual interaction is included. Extensive numerical studies by many authors, mainly of nuclei in the $s d$-shell, have shown that in this case, nearest-neighbor spacing distribution and Mehta-Dyson statistic do agree with GOE predictions whenever the number of nucleons in the shell exceeds three or four. This is true provided the matrix elements of the residual interaction are sufficiently strong to mix configurations pertaining to different single-particle energies within the same major shell. For a review, see Ref. [15]. Recent theoretical studies of the TBRE confirm this picture for the $s d$-shell 16 and show that almost all two-body residual interactions cause chaos. Generalizing this argument we are led to expect that in every major spherical shell, a sufficiently strong residual interaction generically produces chaos. (This statement is not at variance with the existence of regular spectral features near the ground state or like the giant resonances. It relates to spectral statistics, i.e., to the joint properties of a large number of levels). We add in parenthesis that the Skyrme-Hartree-Fock approach [17] or other similar approaches take account of many-body effects in terms of an effective single-particle density functional. In this approach the line separating single-particle and many-particle effects becomes blurred.

The situation is somewhat different in deformed nuclei. For sufficiently strong deformations, the single--particle motion alone may be chaotic, and the single-particle spectrum may obey Wigner-Dyson statistics. But strong deformations occur in the middle between large major shells, so we deal with many valence nucleons. And when we put several or many nucleons into these deformed single-particle states and observe the exclusion principle, we generate a spectrum which by construction is a superposition of several or many GOE spectra. It is known that such superpositions tend rapidly (with increasing nucleon number) towards the Poisson spectrum which is characteristic of integrable motion. This suggests that also in deformed nuclei, the residual interaction is needed to mix the configurations and to generate spectral properties of the Wigner-Dyson type. We are not aware, however, of studies to show under which conditions sufficiently strong mixing is attained.

A different view of chaos in nuclei is offered by the Interacting Boson Model which covers the case of deformed nuclei but also that of nuclei with vibrational spectra etc. That is an effective model which describes the low-energy spec- 
tra of nuclei with many nucleons outside of closed shells, i.e., of nuclei where shell-model calculations are ineffective, in terms of several interacting bosons of angular momenta zero and two. Without any residual interaction between nucleons, the model would not exist. Studies of chaos within this model 18 have shown that chaos is generic except near those parameter values where dynamical symmetries persist.

The available evidence then suggests that except for very special cases where symmetries dominate, it is legitimate to say that almost all forms of the residual interaction cause chaos, and that chaos would not exist without the residual interaction. This view is also supported by the empirical evidence, i.e., the agreement of the spectral fluctuations of the nuclear data ensemble with Wigner-Dyson statistics [19, and the available evidence at lower excitation energies, see Ref. 20] and references therein.

This view sheds new light on attempts to reduce the discrepancy between mass formulas and data by taking into account the residual interaction in some way, either by explicit diagonalization, or by using relations between binding energies of the Garvey-Kelson type or some other local (in $A$ ) information. Several successful such attempts have been published, see, for instance, Refs. 6, 7, 8, 9]. Inasmuch as the residual interaction produces chaos, this success does not contradict the interpretation of the discrepancy in terms of chaotic nuclear motion. The same remark would apply to versions of the energy-density functional theory where the residual interaction is incorporated in the single-particle Hamiltonian.

In some papers 21, 8] chaos in nuclei is interpreted as limiting the accuracy with which spectra or binding energies in nuclei can be calculated. It is claimed that the error estimated in Eq. (11) constitutes a bound on the possible accuracy of dynamical calculations of nuclear masses, and any reduction of this bound is then taken as proof that the discrepancy does not have its origin in chaos. We disagree with this point of view. The spectra of nuclei in the $s d$-shell, for instance, do display chaos but can be calculated without any limitation on the numerical accuracy. This point is discussed in Ref. [16. More generally, it was shown in Ref. 22] that while classical chaos does indeed limit dynamical calculations to a time scale given by the Lyapunov coefficient, this is not true in general for quantum calculations.

In a recent paper 23 it was shown that correlations among binding energies can also be accounted for in the framework of the mean-field approach. So far, this work is restricted to neighboring isotopes differing by a few units in mass number.

In summary, we have interpreted the discrepancy between the nuclear mass formula and nuclear masses in terms of effects due to the residual interaction. Using the two-body random ensemble, we have shown that correlations exist between all binding energies and all separation energies throughout the valley of stability. To the best of our knowledge, this is the first evidence for such wideranging correlations in nuclei. We have argued that chaos in nuclei is generically caused by the residual interaction and does not exist without it. Therefore, we believe that there is no substantial difference between our view and the one taken 
in Ref. 4. There are successful attempts to reduce the discrepancy by taking into account parts of the residual interaction not contained in the semiempirical mass formula in theoretical calculations. Such attempts do not contradict the interpretation of the discrepancy displayed in Ref. [4] in terms of chaotic nuclear motion.

The authors are grateful to T. Papenbrock for helpful discussions.

\section{References}

[1] P. Möller, J. R. Nix, W. D. Myers, and W. J. Swiatecki, Atom. Data Nucl Data Tables 59 (1995) 185.

[2] M. Samyn, S. Goriely, M. Bender, and J. M. Pearson, Phys. Rev. C 70 (2004) 04439.

[3] J. Duflo and A. P. Zuker, Phys. Rev. C 52 (1995) R23.

[4] O. Bohigas and P. Leboeuf, Phys. Rev. Lett. 88 (2002) 092502; ibid. 129903.

[5] M. V. Berry, Proc. R. Society London A 400 (1985) 229.

[6] J. G. Hirsch, A. Frank, and V. Velasquez, Phys. Rev. C 69 (2004) 37304.

[7] J. G. Hirsch, V. Velasquez, and A. Frank, Phys. Lett. B 595 (2004) 231.

[8] J. Barea, A. Frank, J. G. Hirsch, and P. van Isacker, nucl-th/0502038.

[9] V. Velasquez et al., nucl-th/0503073

[10] S. C. Pieper, R. B. Wiringa, Ann. Rev. Nucl. Part. Sci. 51 (2001) 53.

[11] J. B. French and S. S. M. Wong, Phys. Lett. B 33 (1970) 449.

[12] O. Bohigas and J. Flores, Phys. Lett. B 34 (1971) 261.

[13] A. Molinari and H. A. Weidenmüller, Phys. Lett. B 601 (2004) 119.

[14] T. Papenbrock and H. A. Weidenmüller, Phys. Rev. C 73 (2006) 014311.

[15] V. Zelevinsky, B. A. Brown, N. Frazier, and M. Horoi, Phys. Rep. 276 (1996) 85.

[16] T. Papenbrock and H. A. Weidenmüller, Nucl. Phys. A 757 (2005) 422.

[17] G. F. Bertsch, B. Sabbey, and M. Uusnäkki, Phys. Rev. C 71 (2005) 054311 .

[18] Y. Alhassid, N. Whelan, Phys. Rev. C 43 (1991) 2637.

[19] O. Bohigas, R. U. Haq, and A. Pandey, Phys. Rev. Lett. 43 (1982) 1026. 
[20] A. Y. Abul-Magd, H. L. Harney, M. H. Simbel, and H. A. Weidenmüller, Phys. Lett. B 579 (2004) 278.

[21] S. Aberg, Nature 417 (2002) 499.

[22] G. Casati, B. V. Chirikov, I. Guarneri, and D. L. Shepelyansky, Phys. Rev. Lett. 56 (1986) 2437.

[23] H. Olofsson, S. Aberg, O. Bohigas, and P. Leboeuf, Phys. Rev. Lett. 96 (2006) 042502. 